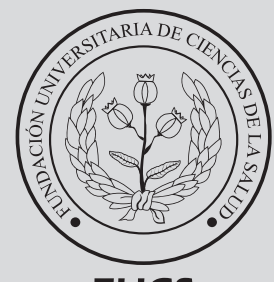

FUCS
Re por

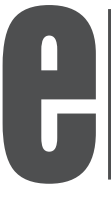

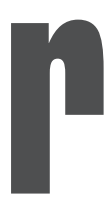
to

de

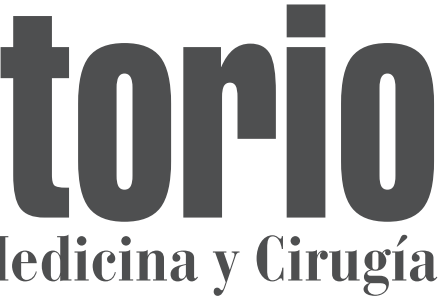

Historia de la medicina

\title{
Crisis epiléptica en la obra Transfiguración de Rafael Sanzio
}

\section{Epileptic seizure depicted in The Transfiguration a work by Raphael Sanzio}

${ }^{a}$ Neurologia, Grupo de investigación en Neurociencia (NEUROS), Universidad del Rosario. Bogotá DC, Colombia.

\section{R E S U M EN}

Entre 1516 y 1520 Rafael Sanzio (1483-1520) realizó la obra Transfiguración. Es un cuadro de gran formato que representa un relato de los evangelios de Lucas, Mateo y Marcos con dos escenarios principales, el primero en la parte superior muestra la transfiguración de Cristo en el Monte Tabor; el segundo en la parte inferior expone la escena de un niño endemoniado que varios apóstoles intentan curar infructuosamente, lo cual solo ocurrió cuando Jesús lo hizo. Esta composición, en otras palabras, muestra un milagro fallido que no es habitual en el arte sacro. Un análisis desde la semiología neurológica permite aseverar que el niño poseído está presentando una crisis epiléptica tónica postural. Este artículo analiza la obra, la semiología neurológica en ella y su relación con la historia de la neurología.

Palabras clave: transfiguración, niño endemoniado, epilepsia, crisis tónica postural.

(C) 2020 Fundación Universitaria de Ciencias de la Salud - FUCS Este es un artículo Open Access bajo la licencia CC BY-NC-ND (http://creativecommons.org/licenses/by-nc-nd/4.0/).

INFORMACIÓN DEL ARTÍCULO

Historia del artículo:

Fecha recibido: marzo 30 de 2020

Fecha aceptado: mayo 11 de 2020
Autor para correspondencia.

Dr. Leonardo Palacios leonardo.palacios@urosario.edu.co
DOI

10.31260/RepertMedCir.01217372.1029 
Raphael Sanzio (1483-1520) painted The Transfiguration between 1516 and 1520. It is a large format painting that illustrates two main scenes as described in the Gospel accounts of Luke, Mathew and Mark. The first scene, in the upper half, shows Christ's transfiguration on Mount Tabor; the lower half, shows the devil-possessed child with the apostles who had attempted unsuccessfully to heal him, but Christ heals the boy. In other words, this composition shows a failed miracle which is unusual in sacred art. Based on a neurological semiology analysis we can assert that the possessed boy is experiencing a tonic postural seizure. This article analyzes the painting, the neurological features depicted in it and its relationship with the history of neurology.

Key words: transfiguration, devil-possessed child, epilepsy, tonic postural seizure.

(C) 2020 Fundación Universitaria de Ciencias de la Salud - FUCS. This is an open access article under the CC BY-NC-ND license (http://creativecommons.org/licenses/by-nc-nd/4.0/).

\section{LA OBRA}

Encargo, elaboración y ubicación actual. La obra Transfiguración fue realizada entre 1516 y 1520 por el maestro Rafael Sanzio (1483-1520) uno de los más importantes pintores del renacimiento italiano. Fue encargada por el cardenal Julio de Medici (1478-1534) futuro Papa Clemente VII, para adornar la catedral de la ciudad francesa de Narbona, hacia la cual sentía especial aprecio por haber recibido allí su obispado en 1515 . Esta fue la última obra realizada por el artista italiano que a su fallecimiento la estaba terminando. Se presume que el cuadro fue terminado por uno de sus discípulos Guilio Romano (1499-1546). Rafael fue velado en El Vaticano a los pies de esta obra, la cual nunca llegó a Francia ya que el cardenal se quedó con el cuadro. Posteriormente lo regaló al convento de San Pedro en Montorio, Roma, donde adornó el altar mayor. En el contexto de las guerras napoleónicas y en virtud del tratado de Tolentin firmado en 1797, el cuadro pasó a pertenecer a Francia, hasta la caída de Napoleón Bonaparte. En 1815 retornó a Italia y pasó a formar parte de la colección de la pinacoteca vaticana, donde se encuentra en la actualidad. ${ }^{1-3}$

\section{DESCRIPCIÓN}

Se trata de una obra (figura 1) de gran formato, cuyas medidas son $405 \times 278$ centímetros, pintada al óleo sobre madera. El cuadro muestra dos episodios relatados en los evangelios de Mateo, Marcos y Lucas. En la parte superior se observa la transfiguración de Cristo en el Monte Tabor (o Hermón, según la tradición) y el segundo y en la inferior el intento infructuoso de algunos de los apóstoles por curar a un niño poseído por el demonio.

El relato de Mateo, que es el más completo, refiere que Jesús llevó a Pedro, Santiago y Juan a un monte elevado y se transfiguró delante de ellos. Como se observa, Jesús aparece vestido de blanco, profundamente iluminado y a sus lados se encuentran Moisés y Elías conversando con él. Luego de la transfiguración los evangelistas mencionados relatan el episodio del endemoniado con algunas variaciones. Los tres se refieren al descenso del Monte, en una las versiones se señala que la multitud salió a recibirlos y que un hombre le habló a Jesús pidiéndole auxilio para que ayudara a su hijo. En otra versión se describe una reunión en que los escribas están discutiendo con la multitud y Jesús se acerca a preguntarles el motivo de la discusión. En el evangelio de Mateo el hombre sale al encuentro y arrodillado ante Jesús le pide que sane a su hijo que es un lunático. El niño es llevado ante Jesús quien expulsa el demonio de él y lo sana. ${ }^{3}$ Este cuadro entonces presenta la transfiguración, el intento infructuoso de varios apóstoles por curar al niño endemoniado, lo cual no ocurriría sino hasta que Jesús lo realizó. Lo interesante de esta obra es que muestra un milagro fallido, lo que no es frecuente en el arte sacro. ${ }^{3}$

EL NIÑO ENDEMONIADO O LUNÁTICO, CONTEXTO HISTÓRICO Y ANÁLISIS A LA LUZ DE LA SEMIOLOGÍA NEUROLÓGICA

La escena que domina la porción inferior de la obra es de un niño "endemoniado" o "lunático". La palabra epilepsia no se utilizaba en ese entonces, al parecer apareció por primera vez en escritos de Avicena de Bagdad (980-1037 dC) y desde entonces fue universalmente adoptado. ${ }^{4,5} \mathrm{La}$

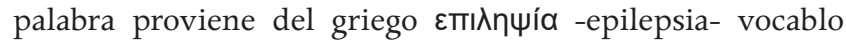

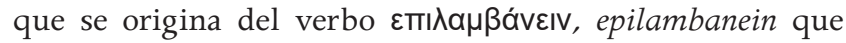
significa sujetar, agarrar o poseer y se refiere a un estado de posesión, sumisión o ataque. ${ }^{6,7}$ El verbo es pasivo y sugiere que una fuerza extraña posee al individuo.,

En cambio al principio de nuestra era solía denominarse como morbus demoniacus -mal demoníaco-, indicando un episodio producto de una posesión diabólica. Esta denominación persistió durante más de mil años y fue 
- Repertorio

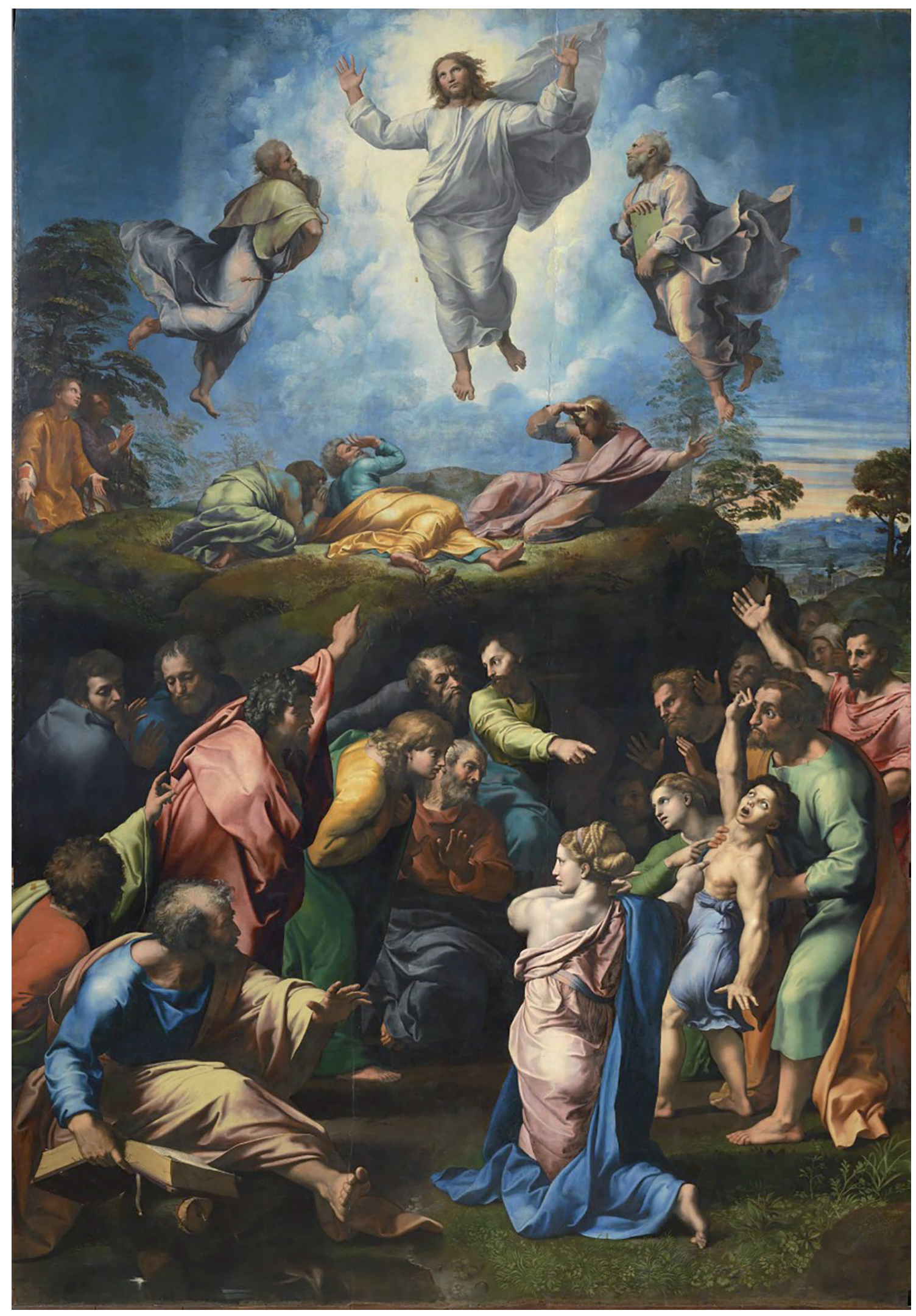

Figura 1. Transfiguración, Rafel Sanzio, Museo del Vaticano, Sala VIII, siglo XVI. ${ }^{1}$ 
motivo de estigmatización y de maltrato hacia las personas que padecían esta entidad. ${ }^{4,8,9}$ La denominación lunática data de la misma época y está relacionada con la idea de que el diablo o enviados de él aparecían en noches de luna llena para poseer sujetos y producir cambios en su comportamiento. El término también se empleaba para referirse a personas con enfermedad mental que eran denominados lunáticos. ${ }^{10,11}$

La crisis que presenta el niño del cuadro ha sido analizada por varios neurólogos y epileptólogos y se considera que corresponde a una crisis tónica postural. Este tipo de actitud corporal se ha observado en crisis que se originan en el lóbulo frontal. ${ }^{12,13}$ En 1986 el epileptólogo alemán Dieter Janz (1920-2016) publicó un artículo sobre esta obra. En él señalaba una posible asociación entre la parte superior, representando la transfiguración, y la inferior en la que se observa el niño presentando la crisis, que a juicio del autor sería una representación simbólica de un evento trascendente. Este aspecto metafísico de la epilepsia representado por Rafael Sanzio, se encuentra también en los pasajes bíblicos que ya fueron citados. La situación del paciente puede asimilarse a la transfiguración a través del sufrimiento, la muerte y la resurrección. ${ }^{14,15}$

\section{CONCLUSIONES}

Se puede afirmar que en la historia de la humanidad el arte y la medicina, en este caso particular la epilepsia, se encuentra entrelazadas de muchas formas y a través de diversas expresiones artísticas. La magnífica obra Transfiguración de Rafael Sanzio es un excelente ejemplo sobre la contribución que las artes aportan en la formación de los profesionales de la salud y enriquecen aspectos fundamentales de nuestra profesión, como el humanismo médico.

\section{AGRADECIMIENTOS}

A la doctora María Catalina Sánchez Martínez asistente de investigación. Grupo de Estudios Sociales de las Ciencias, las Tecnologías y las Profesiones, Universidad del Rosario, por su asesoría en la revisión editorial.

\section{REFERENCIAS}

1. Sanzio R. Transfiguración [Internet]. Italia: Museo del Vaticano, Sala VIII s.XVI; 1516- 1520 [cited 2020 marzo 26]; Recuperado de: http://www.museivaticani.va/content/museivaticani/es/ collezioni/musei/la-pinacoteca/sala-viii---secolo-xvi/raffaellosanzio--trasfigurazione.html\#\&gid=1 \&pid=1
2. Henry T. Rafael Sanzio [Internet]. Madrid, España: Museo del Prado; s.f. [cited 2020 marzo 26]; Recuperado de: https://www. museodelprado.es/aprende/enciclopedia/voz/rafael-sanzio/ cfde10c0-f78c-4280-9ce3-3e7596cabecb.

3. Viñuales J. La Transfiguración de Rafael : un mito plástico. Espacio, Tiempo y Forma Series I-VII. 2000;13:51-83.

4. Lennox W. Epilepsy and Related Disorders. Brain. 1960;83(4):7589. doi: https://doi.org/10.1093/brain/83.4.758

5. Rocha Arrieta LL. La enfermedad que alguna vez fue sagrada. Ciencia. 2005;56(3):7-13.

6. de Villiers JC. A few thoughts on the history of epilepsy. S Afr Med J. 1993;83(3):212-5.

7. Lebrun Y. Language and epilepsy: an introduction. J. Neurolinguistics. 1991;6(4):361-70. doi: https:/doi. org/10.1016/0911-6044(91)90010-G

8. Kottek SS. "Mater puerorum'. A medieval naming for an enigmatic children's disease. Eur J Pediatr. 1981;137(1):75-9. doi: https:// doi.org/10.1007/bf00441174

9. Sterpellone L. El síntoma sagrado. Historias curiosas de la medicina: usos y costumbres de la medicina, desde la antiguendad hasta nuestros días. Barcelona: Ediciones Robinbook; 2009. p. 77-80.

10. Gordon Lennox W, Lennox-Buchthal MA. Paroxysmal disorders. Epilepsy and related disorders. Boston: Little, Brown and Company; 1960. p. 3.

11. Reynolds EH, Trimble MR. Epilepsy, psychiatry, and neurology. Epilepsia. 2009;50 Suppl 3:50-5. doi: https://doi.org/10.1111/ j.1528-1167.2009.02039.x

12. Mann MW. The epileptic seizure and the mystery of death in Christian painting. Epilepsy Behav: E\&B. 2010;17(2):139-46. doi: https://doi.org/10.1016/j.yebeh.2009.10.006

13. Rektor I, Schachter SC, Arzy S, Baloyannis SJ, Bazil C, Brazdil M, et al. Epilepsy, behavior, and art (Epilepsy, Brain, and Mind, part 1). Epilepsy Behav. 2013;28(2):261-82. doi: https://doi.org/10.1016/j. yebeh.2013.03.011

14. Janz D. Epilepsy, viewed metaphysically: an interpretation of the biblical story of the epileptic boy and of Raphael's transfiguration. Epilepsia. 1986;27(4):316-22. doi: https://doi. org/10.1111/j.1528-1157.1986.tb03548.x

15. WattsG. Wolfgang Dietrich Janz. TheLancet. 2017;389(10076):1292. doi: https://doi.org/10.1016/S0140-6736(17)30829-2 